\title{
Amoxicillin/Clavulanic Acid-Induced Agranulocytosis: A Case Report and Review of the Literature
}

\author{
Aristides Armas Villalba $\mathbb{D}^{1}{ }^{1}$ Marya Haq, ${ }^{1}$ Cunxian Zhang, ${ }^{2,3}$ and Jinen Thakkar ${ }^{1}$ \\ ${ }^{1}$ Internal Medicine Department, Kent County Hospital, Warren Alpert Medical School of Brown University, Providence, RI, USA \\ ${ }^{2}$ Associate Professor of Pathology and Laboratory Medicine, Brown University, Providence, RI, USA \\ ${ }^{3}$ Chief of Pathology, Kent County Hospital, Warwick, RI, USA
}

Correspondence should be addressed to Aristides Armas Villalba; aristides_armas_villalba@brown.edu

Received 2 April 2019; Revised 22 August 2019; Accepted 1 October 2019; Published 23 October 2019

Academic Editor: Kazunori Nakase

Copyright (C) 2019 Aristides Armas Villalba et al. This is an open access article distributed under the Creative Commons Attribution License, which permits unrestricted use, distribution, and reproduction in any medium, provided the original work is properly cited.

\begin{abstract}
Amoxicillin/clavulanic acid is one of the widely prescribed antibiotics in the outpatient setting giving excellent antimicrobial coverage and relatively safe profile in terms of adverse reactions. Gastrointestinal and cutaneous reactions are among the commonly reported. Our goal is to describe a case of agranulocytosis induced by amoxicillin/clavulanic acid in a previously healthy patient and review prior reports with similar presentation.
\end{abstract}

\section{Introduction}

According to the Center for Disease Control and Prevention (CDC), 269.4 millions of antibiotic prescriptions were ordered in the outpatient setting within the United States of America in 2015. Amoxicillin is the most commonly prescribed antibiotic, and amoxicillin-clavulanate is the third most commonly prescribed [1].

There are drugs classically described as the potential cause of neutropenia or agranulocytosis, but amoxicillin/ clavulanate is not included in the list. Since the development of the drug, the most common side effect or adverse reaction reported is diarrhea.

Here, we present a case about amoxicillin/clavulanateinduced agranulocytosis and review of the literature.

\section{Case Report}

A 49-year-old male patient without a significant past medical history presented to hospital with complaints of diarrhea. Five days prior to presentation, he developed a sore throat and consulted a local urgent care center where rapid influenza and streptococcal antigen testing were completed. Despite the negative results of both of these tests, he was prescribed amoxicillin/clavulanate (875/125 mg twice a day, day 5 on presentation to the hospital).

His symptoms persisted and progressed, with the addition of watery diarrhea to his complaints, at which point he presented to the emergency department. Initial physical exam was significant for a temperature of $38.7^{\circ} \mathrm{C}\left(101.7^{\circ} \mathrm{F}\right)$, with other vital signs within normal limits. Skin exam demonstrated a generalized macular and erythematous exanthema. The pharynx was found to be erythematous but without exudates, and chest auscultation demonstrated bilateral wheezing. The remainder of the physical exam was unremarkable.

Initial laboratory data reported hemoglobin $14.0 \mathrm{~g} / \mathrm{dL}$, hematocrit $40.6 \%$, platelet count of $159 \times 10^{3} / \mathrm{mm}^{3}$, and white blood cell count of $500 / \mathrm{mm}^{3}$. Considering the fever, physical examination and degree of neutropenia, the patient was admitted to the hospital with diagnosis of febrile neutropenia and started on broad-spectrum antibiotics immediately (vancomycin and piperacillin-tazobactam).

At the same time, blood cultures were drawn and the patient was started on filgrastim (granulocyte-colony stimulating factor) and continued on a daily basis. When he remained febrile and profoundly leukopenic, the hematology department was consulted for bone marrow aspirate and 
biopsy. On day three, marrow examination was done and reported a 40-60\% cellularity with myeloid hypoplasia (myeloid to erythroid precursor ratio of $0.5: 1$ ) and increased blasts (Figure 1).

Despite a slowly rising leukocyte count, he continued to spike fevers for the first seven days of admission; on day nine of admission, his leukocyte count had normalized (Figure 2). Throughout the stay in hospital, extensive work-up for infectious and immunologic causes of agranulocytosis (Table 1) was done and revealed only a positive IgG result against parvovirus B19. Since the diagnosis was still a challenge, skin biopsy of the aforementioned rash was performed and reported perivascular and periadnexal lymphoplasmacytic inflammation.

His clinical condition continued to improve, with stable vital signs including lack of recurrence of fevers for the last four days of his inpatient stay, and as there were no further indications for either antibiotics or filgrastim, he was discharged home on day eleven of his hospital stay.

One week after discharge, he was seen in follow-up by his hematologist, with continued demonstration of a complete blood count within normal limits and no further concerning signs or symptoms.

\section{Discussion}

Classically, neutropenia is defined as an absolute blood neutrophil count less than two standard deviations below the normal mean, and agranulocytosis is often used to define severe neutropenia (below $0.5 \times 10^{9}$ cells/liter) [2]. The causes of neutropenia can be classified as a disorder in the production and distribution or turnover of neutrophils.

In terms of duration, neutropenia can be classified as transient or chronic, with three months being the cutoff point. The most common etiology of transient neutropenia is a viral infection [3].

Several studies have studied the incidence of drug-induced neutropenia, estimating it anywhere between 1.1 and 15.4 cases per million population around the world per year [4-6].

Interestingly, despite an extensive infectious work-up, no infectious etiology was identified in this patient with acute onset agranulocytosis or neutropenia. He did present to the emergency department six days after the onset of symptoms, but most infectious causes of agranulocytosis do not typically cause the persistent marrow suppression seen in this patient despite resolution of his symptoms.

Taking the extensive work-up, the presence of a maculopapular rash and the temporal association with exposure to amoxicillin-clavulanate, we concluded that his presentation was consistent with amoxicillin-clavulanate-induced agranulocytosis. On top of that, ferritin and C-reactice protein levels were found to be elevated, well know acute phase reactants that can be triggered by immune processes.

There are well-described nonchemotherapeutic medications associated with agranulocytosis, and the term is known as idiosyncratic drug-induced agranulocytosis.

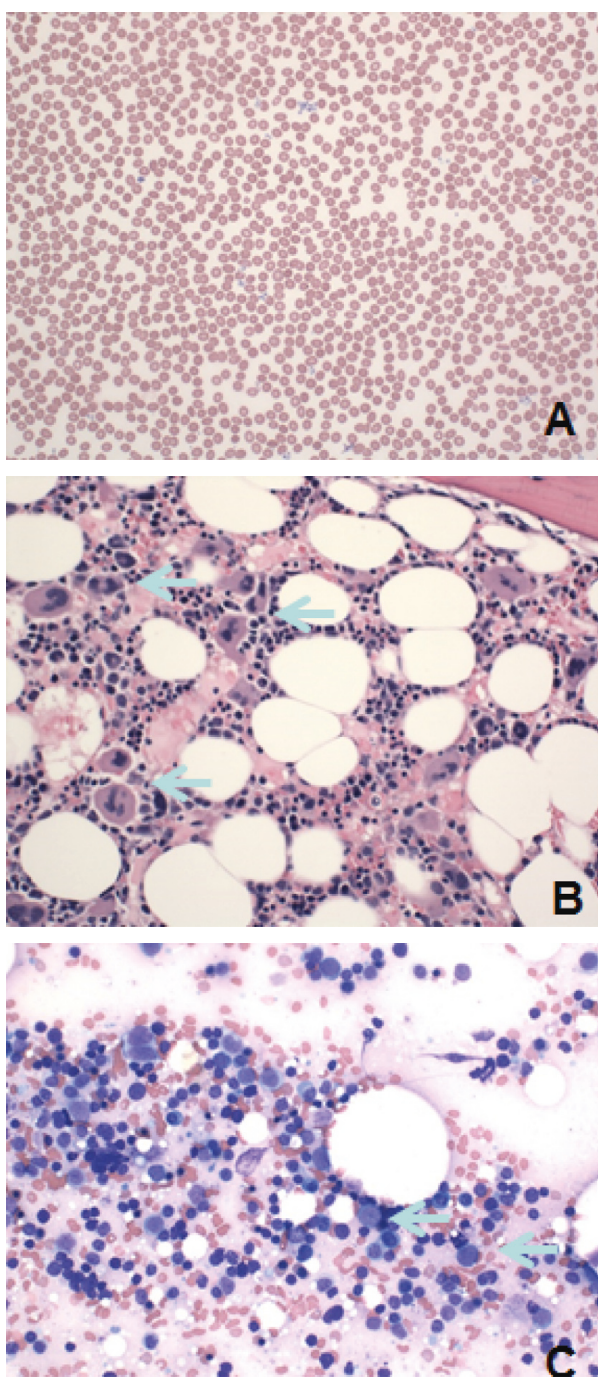

Figure 1: Peripheral blood smear and bone marrow studies. (a) Peripheral blood smear shows severe neutropenia with no neutrophils seen in this field (hematoxylin and eosin stain; $\times 400$ ), (b) bone marrow biopsy with normal cellularity, increased megakaryocytes (blue arrows), and severe decrease in neutrophils (hematoxylin and eosin stain; $\times 400$ ), and (c) bone marrow smear displays severely decreased segmented granulocytes and increased blasts (blue arrows; Wright-Giemsa stain $\times 400$ ).

There are only two case reports associating amoxicillinclavulanate with agranulocytosis, but unfortunately the investigations were not as thorough as our patient had, leading to multiple questions about whether it truly was the drug that caused the hematologic findings [5].

Briefly, the pathogenesis of the disease has been attributed to direct "toxic" effect of the medication (or a metabolite) and recently has been described an immunemediated process. A widely accepted hypothesis explains that the drug can activate the immune system via haptens that later will induce direct damage to the cell or bone marrow itself $[7,8]$.

Our approach to this patient's management was to first exclude infections as a possible etiology, and the extensive workup can be seen in Table 1. In the meantime, broad-spectrum 


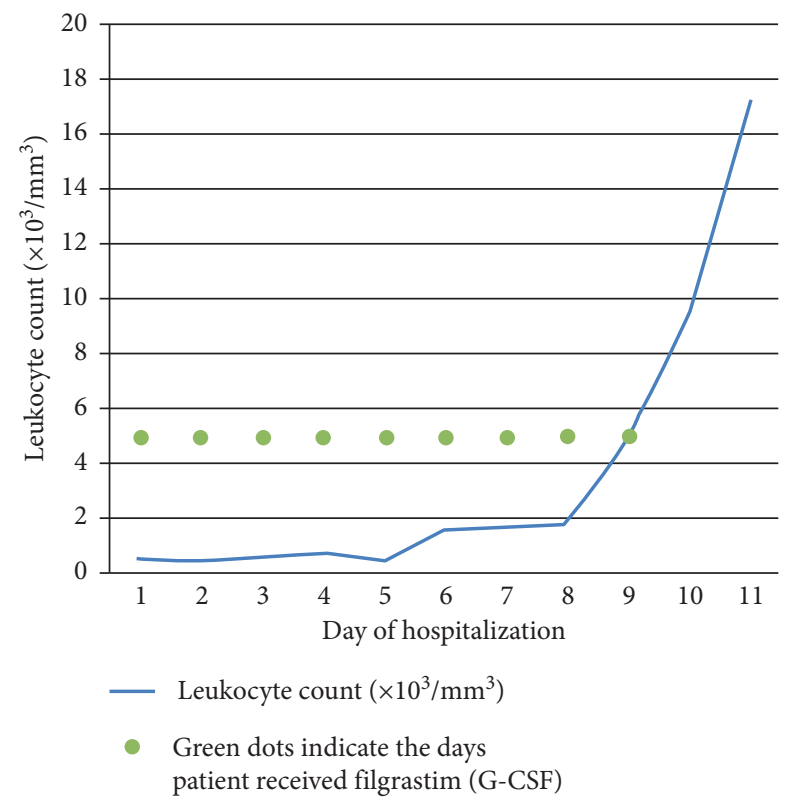

Figure 2: Daily leukocyte trend throughout the admission in the hospital.

TABLE 1: Infectious and immunologic work-up performed during the admission.

\begin{tabular}{|c|c|c|}
\hline Test & Result & Comments \\
\hline Chest X-ray & Normal examination & \\
\hline CT chest, abdomen, and pelvis & Unremarkable & Simple cysts in the left kidney. \\
\hline Blood cultures & Negative & Performed on three different days (two sets each) \\
\hline Stool analysis & C. albicans isolated in the culture & Negative for bacteria, ova, and parasites. \\
\hline Influenza rapid diagnostic test & Negative & \\
\hline Rapid strep test & Negative & \\
\hline Mononucleosis spot test & Negative & IgM heterophile antibody \\
\hline Urine analysis & Negative for infection & \\
\hline Blood parasites & Negative & \\
\hline Viral hepatitis & Negative & Hepatitis A, B, and C. \\
\hline Cytomegalovirus & Negative & IgG and IgM \\
\hline Human immunodeficiency virus & Negative & Elisa and PCR \\
\hline Parvovirus B19 & IgG positive & IgM negative \\
\hline Babesiosis panel & Negative & PCR for B. microti, B. duncani, and B. divergens. \\
\hline T. pallidum antibodies & Negative & \\
\hline Respiratory virus culture & Negative & Reported after 14 days of incubation \\
\hline Fungitell & Negative & \\
\hline B. burgdorferi & Negative & $\operatorname{IgG}$ and $\operatorname{IgM}$ \\
\hline Rheumatoid factor & Negative & \\
\hline Anti-nuclear antibodies & Negative & \\
\hline Ferritin & $615 \mathrm{ng} / \mathrm{mL}$ & Normal values from 18 to $270 \mathrm{ng} / \mathrm{mL}$ \\
\hline Procalcitonin & $0.12 \mu \mathrm{g} / \mathrm{mL}$ & Normal values from 0 to $0.26 \mu \mathrm{g} / \mathrm{mL}$ \\
\hline C3 level & $138 \mathrm{IU}$ & Normal values from 88 to $200 \mathrm{IU}$ \\
\hline C4 level & $37.2 \mathrm{IU}$ & Normal values from 16 to $47 \mathrm{IU}$ \\
\hline C-reactive protein & $17.4 \mathrm{mg} / \mathrm{dL}$ & Upper limit of normal $0.5 \mathrm{mg} / \mathrm{dL}$ \\
\hline
\end{tabular}

antibiotics and granulocyte-colony stimulating factor were administered to the patient as part of the treatment for neutropenic fever.

The patient continued to be severely neutropenic despite multiple treatments with granulocyte-colony stimulating factor (G-CSF). There are several hypotheses proposed to explain the phenomenon: the first one is an immune-mediated reaction after the patient is exposed to the drug and the second one, the drug itself inducing a toxic microenvironment in the marrow that inhibits the production of new cells [9].

Four days after G-CSF was started, the bone marrow was still not responding and bone marrow biopsy and aspirate was performed. The pathology department reported $40-60 \%$ cellularity with marked myeloid hypoplasia. At the same time, skin biopsy was taken as well for further understanding 
of the rash and reported perivascular and periadnexal lymphoplasmacytic inflammation. Both of these findings are consistent the with drug-related reaction.

We conclude that over the years, amoxicillin-clavulanate has demonstrated to be a safe drug that has been widely used. Despite the two prior reports and ours, prescription must be continued if there is a formal indication in the appropriate patient to avoid harming the patient and reducing antibiotic resistance rates as much as possible.

\section{Conflicts of Interest}

The authors declare that they have no conflicts of interest.

\section{Authors' Contributions}

All authors contributed equally to the development of the manuscript.

\section{References}

[1] CDC Centers for Disease Control and Prevention, OutpatientAntibiotic Prescriptions-United States, CDC Centers for Disease Control and Prevention, Atlanta, GA, USA, 2015, https://www.cdc.gov/antibiotic-use/community/pdfs/ Annual-Report-2015.pdf.

[2] D. C. Dale and K. Welte, "Neutropenia and neutrophilia," in Williams Hematology, 9E, K. Kaushansky, M. A. Lichtman, J. T. Prchal et al., Eds., McGraw-Hill, New York, NY, USA, 2018, http://accessmedicine.mhmedical.com/content.aspx?bookid=1581 \&sectionid $=101238861$.

[3] P. E. Newburger and D. C. Dale, "Evaluation and management of patients with isolated neutropenia," Seminars in Hematology, vol. 50, no. 3, pp. 198-206, 2013.

[4] B. L. Strom, J. L. Carson, R. Schinnar et al., "Descriptive epidemiology of agranulocytosis," Archives of Internal Medicine, vol. 152, no. 7, pp. 1475-1480, 1992.

[5] N. Spetrino, M. Arazzi, G. Di Fulvio et al., "Severe neutropenia during treatment with amoxicillin/clavulanic acid in a patient on regular hemodialysis treatment," Giornale Italiano di Nefrologia, vol. 33, no. 6, 2016.

[6] D. W. Kaufman, J. P. Kelly, S. Issaragrisil et al., "Relative incidence of agranulocytosis and aplastic anemia," American Journal of Hematology, vol. 81, no. 1, pp. 65-67, 2006.

[7] B. R. Curtis, "Non-chemotherapy drug-induced neutropenia: key points to manage the challenges," Hematology, vol. 2017, no. 1, pp. 187-193, 2017.

[8] D. Tesfa, M. Keisu, and J. Palmblad, "Idiosyncratic drug-induced agranulocytosis: possible mechanisms and management," American Journal of Hematology, vol. 84, no. 7, pp. 428-434, 2009.

[9] A. Johnston and J. Uetrecht, "Current understanding of the mechanisms of idiosyncratic drug-induced agranulocytosis," Expert Opinion on Drug Metabolism \& Toxicology, vol. 11, no. 2, pp. 243-257, 2015. 


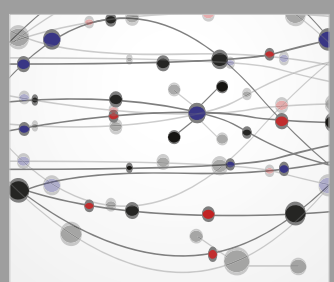

The Scientific World Journal
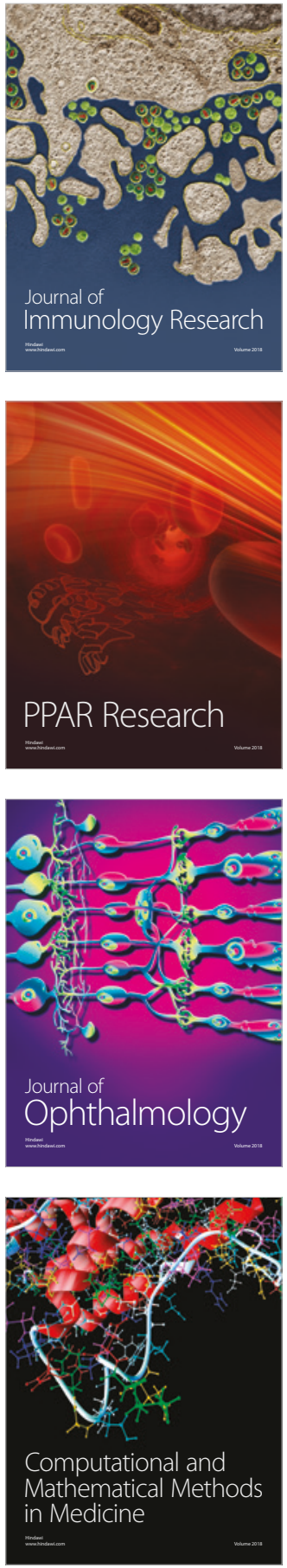

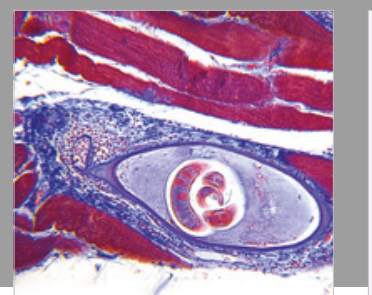

Gastroenterology Research and Practice

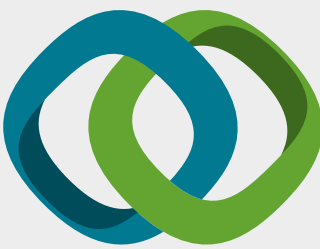

\section{Hindawi}

Submit your manuscripts at

www.hindawi.com
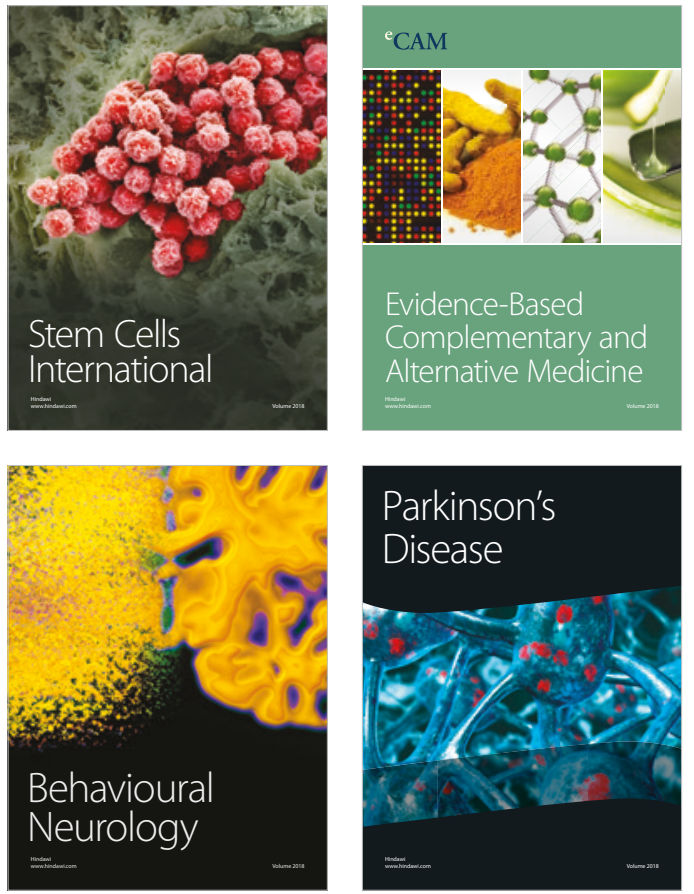

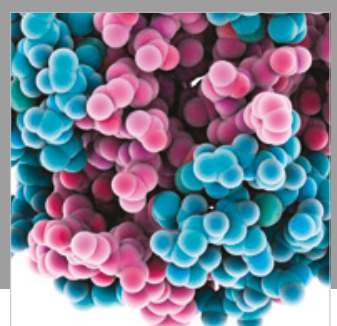

ournal of

Diabetes Research

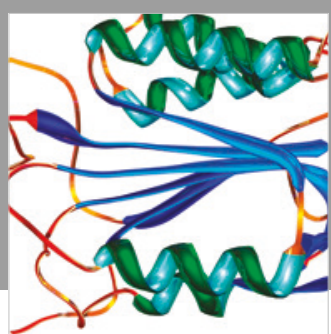

Disease Markers
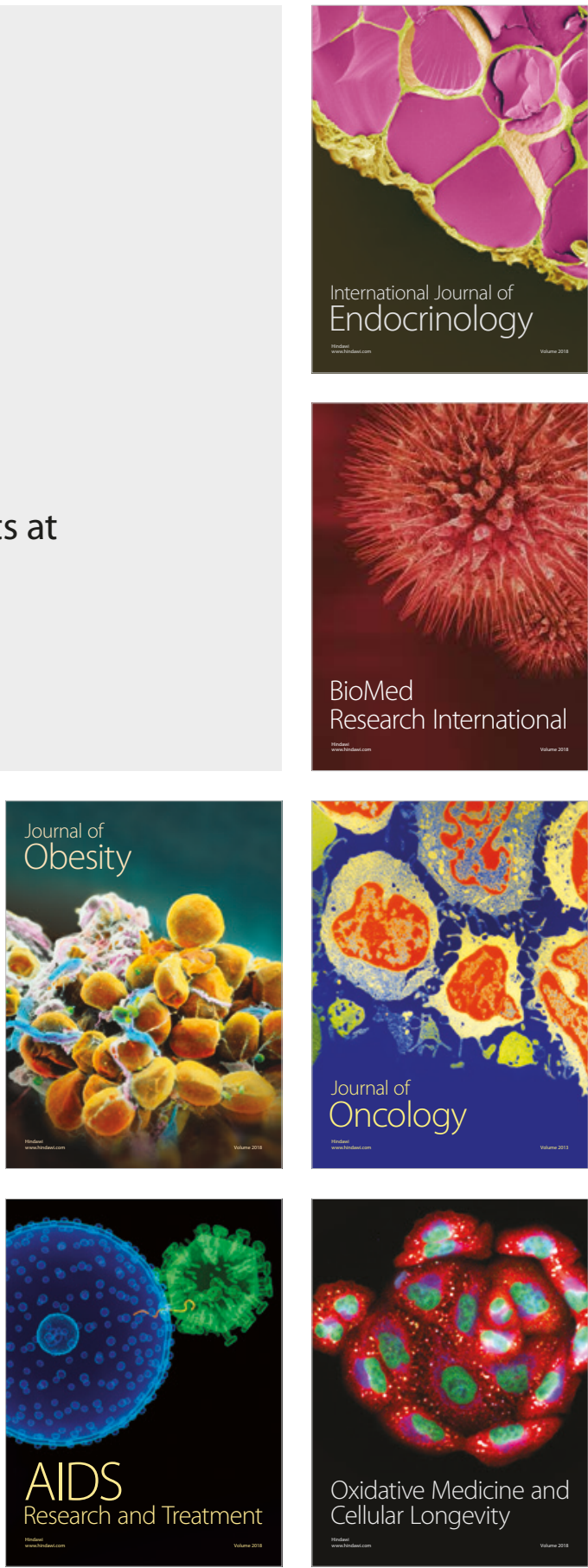